Original Article

\title{
Possible predictive formulas for quantitative and time-based estimation of muscle strength during motion
}

\author{
Umi Matsumura, RPT ${ }^{1)}$, Ayana Kai, RPT ${ }^{2)}$, Miku Numata, RPT ${ }^{3)}$, Yeonghee Lee, RPT ${ }^{1)}$, \\ Shimpei Yamamoto, RPT ${ }^{1)}$, Toshiya Tsurusaki, PhD, $\mathrm{RPT}^{4)^{*}}$ \\ 1) Health Sciences, Nagasaki University Graduate School of Biomedical Sciences, Japan \\ 2) Department of Rehabilitation, Kuroki Memorial Hospital, Japan \\ 3) Department of Rehabilitation, Nagasaki North Hospital, Japan \\ 4) Institute of Biomedical Sciences, Nagasaki University: 1-7-1 Sakamoto, Nagasaki, \\ Nagasaki 852-8520, Japan
}

\begin{abstract}
Purpose] To examine the validity of the predictive formulas based on the angle information of the segment center of mass and moments of inertia, and to propose a joint moment estimation method. [Participants and Methods] Twenty nine young healthy adults were divided into two groups: the Creation group (20 adults) was needed to create the prediction formulas, and the Verification group ( 9 adults) was needed to verify the formulas. By monitoring the Creation group, the angular information from inertial motion sensors and moments of inertia of each limb were used to estimate actual ankle joint moment and knee joint moment. Thereafter, the actual joint moments was derived from the Verification group and compared to the predicted values via Pearson correlations. [Results] Good to excellent correlations were obtained between the actual joint moments of the two groups for most of the motions. [Conclusion] It is suggested that the predictive formulas created from the angle information of the segment center of mass and moments of inertia can be used for an approximate estimation of the lower limb joint moments in the sagittal plane and more clinically useful tools need to be considered in the future.

Key words: Joint moment, Prediction formulas, Muscle strength
\end{abstract}

(This article was submitted Aug. 6, 2019, and was accepted Oct. 17, 2019)

\section{INTRODUCTION}

Muscle strength measurements are essential for determining therapeutic interventions and functional prognosis related to physical therapy ${ }^{1}$. Manual Muscle Testing (MMT) or Hand-Held Dynamometers (HHD) are commonly used for strength assessment ${ }^{2}$. MMT is subjective and can vary with the physical strength of the examiner ${ }^{3)}$. Also, HHD can cause an error depending on the muscle to be assessed and the measurement position ${ }^{4}$. Furthermore, these assessments are limited by measurement of maximum muscle strength that a specific muscle group exerts during isometric contractions in a fixed posture.

Physical therapy, which maintains, restores, and improves movement and activity ${ }^{5}$, would be optimized by evaluations of muscle strength exerted during motions. Initially, motions can be determined by movements that move in a particular manner over time ${ }^{6)}$ and most human movements are rotational of a segment around a joint. Joint moment, defined as the force required at a particular time, can not only be used to reflect muscle force during motions but also the timing of the exertion of muscle strength. For example, the joint moments during walking of patients with knee osteoarthritis differs not only in peak value but also in its timing depending on disease severity ${ }^{7}$. Also, joint moments during moving the seat off in patients with hip osteoarthritis differ as well ${ }^{8}$. Therefore, quantitative and time-based measures of joint moments are necessary in physical

*Corresponding author. Toshiya Tsurusaki (E-mail: toshiya@nagasaki-u.ac.jp)

(C2020 The Society of Physical Therapy Science. Published by IPEC Inc.

(c) (1) $\odot$ This is an open-access article distributed under the terms of the Creative Commons Attribution Non-Commercial No Derivatives cc) 
Table 1. Number of parameters

\begin{tabular}{lccc}
\hline \multicolumn{1}{c}{ Rigid link model } & 5-Link & 4-Link & 3-Link \\
\hline Number of parameters & 58 & 45 & 32 \\
Number of sensors & 7 & 6 & 5 \\
\hline
\end{tabular}

therapy. Currently, the most common methods to measure of joint moments are combining three dimensional motion analysis system with force plate analyses ${ }^{9}$. However, this apparatus is too expensive, requires considerable space and thus is limited to use in clinics ${ }^{10)}$.

Kodama et al. ${ }^{10)}$ have been suggested the way to estimate joint moments of squat and sit-to-stand by using the wearable inertial motion sensors. Their estimation applied the segment parameters calculated based on inertial properties of body segments ${ }^{11)}$ and the segment inclination angles calculated from the motion sensors to Newton's equation of motion. The five-link model and the four-link model that the trunk was divided at the highest point of the iliac crest showed average correlation coefficients of about 0.98 for knee joint and about 0.80 for ankle joint. Even the three-link model that consisted of head, arm and trunk (HAT), Thigh, and Shank showed average correlation coefficients of about 0.95 for knee joint and about 0.60 for ankle joint. However, not only the vertical but also the horizontal factors have to consider and various parameters are necessary in order to solve the equation (Table 1). In addition, it must be applied to the equation one by one, which has a difficulty to generalize. Thus, it can be still difficult to use in busy clinics.

Clinical prediction models are commonly developed to facilitate diagnostic or prognostic probability estimations in daily medical practice ${ }^{12}$. A user-friendly modality is necessary for the use of such formulas and nomograms to become a widespread part of routine practice ${ }^{13)}$. To the best of our knowledge, a prediction model for joint moments has not been developed yet. Joint moment can be calculated from angular acceleration and moment of inertia, although it is well known that joint moment varies with the changing joint angle ${ }^{14)}$. In addition, accelerations of the segment Center of Mass (CoM) position are crucial to calculate joint reaction forces. For these reasons, it is postulated that the prediction formulas for joint moments could be created from the angle information of the segment CoM and moments of inertia. Only the vertical components are utilized for the measurement of joint moments in sagittal plane for the purpose of pursuing the simplicity rather than strict accuracy. Therefore, the purpose of this study was to examine the validity of the prediction formulas created from angle information of the segment CoM and moments of inertia, and to propose an estimation method for joint moments.

\section{PARTICIPANTS AND METHODS}

Twenty-nine (15 males and 14 females) young healthy were analyzed. Individuals were excluded if they had orthopedic conditions, skin abnormalities, or were not able to provide consent. Participants were assigned randomly to two groups: the Creation group to create the prediction formulas of joint moments and the Verification group to verify the formulas. Twenty adults, consisting of 10 males and females (mean age: $21.1 \pm 1.0$ years, mean height: $166 \pm 7.0 \mathrm{~cm}$, mean weight: $60.8 \pm 7.1 \mathrm{~kg}$ ) participated in the Creation group and nine adults, consisting of 5 males and 4 females (mean age: $20.4 \pm 0.8$ years, mean height: $165 \pm 6.0 \mathrm{~cm}$, mean weight: $54.7 \pm 6.5 \mathrm{~kg}$ ) participated in the Verification group. After full explanation of the study, informed written consent was obtained. The study was approved by the Ethics Committee of the Graduate School of Medical Facilities, Nagasaki University (Approved number 18061429).

All the participants performed half squat, knee flexion and extension while standing on one leg, and walking. Participants were instructed to position the trunk vertically over the hip joints ${ }^{15)}$. All motions were measured three times at three different speeds except walking. One-minute rest was taken every one measurement to minimize the effect of fatigue. During walking, participants crossed the force plates starting with their right foot $1 \mathrm{~m}$ from its entry.

Three Wearable inertial motion sensors (LP-WSD1101-OA Ver.1.0.0, $1,000 \mathrm{~Hz}$, LOGICAL PRODUCT, Fukuoka, Japan) were attached to the outside of right limbs by using double-sided adhesive tape ${ }^{10)}$ (Fig. 1). Wearable inertial motion sensors, often consist of tri-axial accelerometer, gyroscopes and magnetic sensors ${ }^{16)}$ can measure angular velocity and have a high accuracy in calculating lower limb segmental angles during motions ${ }^{10,17)}$. The sensors were oriented to have its sensitive axis of rotation perpendicular to the sagittal plane of motion ${ }^{18)}$ in order to obtain the angular information in the segment CoM position. Attachment positions were as follows based on the positions of the approximate each

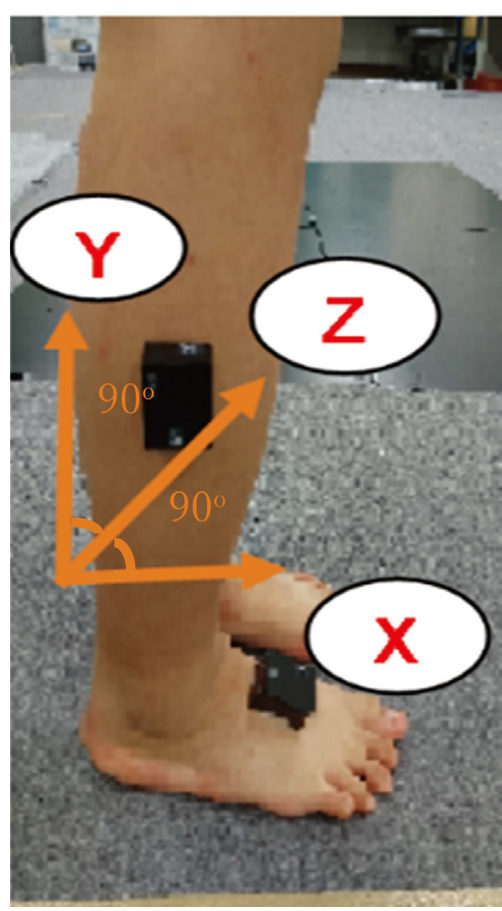

Fig. 1. Wearable inertial motion sensors 
Table 2. Estimation formulas of moment of inertia (Ae et al. 1992)

\begin{tabular}{lc}
\hline \multicolumn{1}{c}{ Gender } & Moment of inertia \\
Male & $\mathrm{I}_{1}=-350.3+418.3 \times \mathrm{L}_{1}+6.6 \times \mathrm{W}$ \\
& $\mathrm{I}_{2}=-62.8+104.7 \times \mathrm{L}_{2}+1.1 \times \mathrm{W}$ \\
$\mathrm{I}_{3}=-41.0+228.1 \times \mathrm{L}_{3}+0.01 \times \mathrm{W}$ \\
$\mathrm{I}_{1}=-262.1-14.1 \times \mathrm{L}_{1}+9.0 \times \mathrm{W}$ \\
Female & $\mathrm{I}_{2}=-46.4+67.1 \times \mathrm{L}_{2}+1.2 \times \mathrm{W}$ \\
& $\mathrm{I}_{3}=32.2+153.7 \times \mathrm{L}_{3}+0.16 \times \mathrm{W}$ \\
\hline I=moment of inertia $\left(\mathrm{kgcm}^{2}\right), 1=$ Thigh, $2=$ Shank, 3=Foot, L: length \\
of the segment $(\mathrm{m}) ; \mathrm{W}$ : weight $(\mathrm{kg})$.
\end{tabular}

segment CoM position ${ }^{11)}$ : Thigh: midway between greater trochanter and lateral knee joint space; Shank: midway between lateral knee joint space and lateral malleolus, Foot: center of dorsum of foot.

The three-dimensional motion analysis system equipped with 16 cameras and four force plates (DF5UM034A-1, 100 Hz, ANIMA, Tokyo, Japan) were used to measure actual joint moments. Reflection markers were attached to both anterior superior iliac spines, greater trochanters, lateral knee joint spaces, lateral malleoli, fifth metatarsal bones. These were synchronized to the motion sensors by using the wireless 8ch logger (LP-WSD1311-OA Ver1.1.0, LOGICAL PRODUCT).

All data were resampled to $1 / 30$ seconds on the time axis by taking an average to reduce the high-frequency noise and to avoid aliasing of the sampling signal ${ }^{19}$ ). Analysis was done on sagittal plane and regarded as the rigid link model consisting of Thigh, Shank and Foot. The angular velocities $v_{j}(\mathrm{deg} / \mathrm{s})$ were integrated with time displacement $\mathrm{dt}(\mathrm{s})$ by using the trapezoidal approximation to calculate the angle of limbs $\theta_{\mathrm{j}}(\mathrm{deg})$.

j=1-3 1=Thigh, $2=$ Shank, $3=$ Foot $\theta_{\mathrm{j}}=\int \mathrm{v}_{\mathrm{j}}(\mathrm{t}) \times \mathrm{dt}$

The lineal resetting mechanism was applied by weighting linearly during integration in order to remove measurement error due to noise and drift ${ }^{20)}$. The calculated angles were converted to the angular accelerations $\mathrm{a}_{\mathrm{j}}\left(\mathrm{deg} / \mathrm{s}^{2}\right)$ by doubly differentiating.

$$
a_{j}=d^{2} \theta_{j} / d^{2}
$$

The moments of inertia of the segments $\mathrm{I}_{\mathrm{j}}\left(\mathrm{kgcm}^{2}\right)$ were estimated by substituting the body weight $\mathrm{W}(\mathrm{kg})$ and the partial lengths of limbs $\mathrm{L}_{\mathrm{j}}(\mathrm{m})$ into the estimation formula (Table 2) created by Ae et al ${ }^{11)}$. Partial lengths were measured as follows: Thigh-greater trochanter to lateral knee joint space; Shank-lateral knee joint space to lateral malleolus; Foot-lateral malleolus to fifth metatarsal bone.

Statistical analysis was performed using JMP Pro 14 (SAS Institute Inc.). Stepwise-multi-regression analysis was performed to create the prediction formulas from the Creation group data. Actual Ankle Joint Moment (AJM) and Knee Joint Moment (KJM) were targeted variables whereas angular information and moments of inertia of each segment were explanatory variables. The foot rotation was accompanied by shank rotation at the motions under no load. Therefore, the explanatory variables did not include the foot items from knee flexion during single leg stance or swing phase during walking. The significance level for adopting the coefficients was $\mathrm{p}<0.05$. Joint moments were estimated by inserting each explanatory variable of the Verification group into the formulas determined from the data of the Created group. Predicted and actual joint moments were compared by Pearson correlation analyses. Correlations were rated according to Portney and Watkins ${ }^{21)}$ : little or no relationship if $\leq 0.25$; fair if 0.25 to 0.50 ; moderate to good if 0.50 to 0.75 and good to excellent if $\geq 0.75$.

\section{RESULTS}

Angular information obtained from the motion sensors are shown as the range between maximum and minimum values (Table 3). The angular accelerations were quite large relative to the angles in every motion. Only coefficients of the prediction formulas that were included in the regression analysis are listed in Table 4. Table 5 showed the peak of each direction and the correlations between predicted and actual joint moments. Joint moments represented plus toward extension and minus toward flexion. The peak of the predicted moments often differed from the actual ones. Though AJM had little correlation and differed within each individual $\mathrm{R}=0.34$ (0.39), KJM had good to excellent correlations with little differences among individuals $\mathrm{R}=0.93$ (0.03) during Half squat. Also, AJM and KJM showed good to excellent correlations in Knee flexion-extension during single leg stance: Knee flexion, AJM; R=0.89 (0.03), KJM; 0.78 (0.12) and Knee extension, AJM; R=0.94 (0.02), KJM; 0.94 (0.02). Furthermore, walking, a moving motion, also moderate to good correlations for both AJM and KJM, AJM; 0.79 (0.08), KJM; $0.51(0.15)$.

\section{DISCUSSION}

This study aimed to examine the validity of the prediction formulas created from angle information of the segment CoM and moments of inertia, and to propose an estimation method for joint moments in clinics. Since the equations of moments 
Table 3. Range of angular information

\begin{tabular}{cllccc}
\hline \multicolumn{1}{c}{ Angular information } & Segment & Half squat & Knee flexion & Knee extension & Walking \\
\hline \multirow{3}{*}{ Angle (deg) } & Thigh & $48(15)$ & $20(7)$ & $83(18)$ & $39(8)$ \\
& Shank & $37(8)$ & $111(17)$ & $125(23)$ & $77(7)$ \\
& Foot & $7(4)$ & $72(18)$ & $77(19)$ & $52(10)$ \\
\multirow{2}{*}{ Angular acceleration $\left(\mathrm{deg} / \mathrm{s}^{2}\right)$} & Thigh & $2,588(2,075)$ & $3,193(2,274)$ & $4,872(3,860)$ & $7,821(4,288)$ \\
& Shank & $1,266(756)$ & $6,160(4,032)$ & $6,842(4,377)$ & $9,408(2,821)$ \\
& Foot & $1,303(1,141)$ & $8,721(4,470)$ & $8,923(4,399)$ & $14,432(4,429)$ \\
\hline
\end{tabular}

Range was represented difference between maximum and minimum values. Visible values showed mean (SD).

Table 4. Coefficient of prediction formulas

\begin{tabular}{|c|c|c|c|c|c|c|c|c|c|c|c|}
\hline \multirow{3}{*}{$\begin{array}{l}\text { Explanation } \\
\text { variables }\end{array}$} & \multirow{3}{*}{ Segment } & \multirow{2}{*}{\multicolumn{2}{|c|}{ Half squat }} & \multirow{2}{*}{\multicolumn{2}{|c|}{ Knee flexion }} & \multirow{2}{*}{\multicolumn{2}{|c|}{ Knee extension }} & \multicolumn{4}{|c|}{ Walking } \\
\hline & & & & & & & & \multicolumn{2}{|c|}{ Stance phase } & \multicolumn{2}{|c|}{ Swing phase } \\
\hline & & AJM & KJM & AJM & KJM & AJM & KJM & AJM & KJM & AJM & KJM \\
\hline \multirow{3}{*}{ Angle } & Thigh & 0.33 & 0.42 & 0.86 & 2.41 & 2.60 & 2.57 & -1.25 & -0.24 & & 0.14 \\
\hline & Shank & 0.63 & -0.77 & 1.83 & -0.09 & 3.21 & 0.09 & -0.30 & 0.13 & $3.0 \times 10^{-3}$ & -0.08 \\
\hline & Foot & -0.33 & -0.42 & & & & & 0.17 & 0.12 & & \\
\hline \multirow{2}{*}{$\begin{array}{l}\text { Angular } \\
\text { acceleration }\end{array}$} & Thigh & $1.0 \times 10^{-3}$ & $-1.0 \times 10^{-3}$ & 0.01 & & $3.0 \times 10^{-3}$ & $2.0 \times 10^{-3}$ & -0.01 & & $-4.0 \times 10^{-5}$ & $-1.0 \times 10^{-3}$ \\
\hline & $\begin{array}{l}\text { Shank } \\
\text { Foot }\end{array}$ & $\begin{array}{l}-5.0 \times 10^{-3} \\
-3.0 \times 10^{-3}\end{array}$ & 0.01 & 0.01 & $3.0 \times 10^{-3}$ & $4.0 \times 10^{-3}$ & 0.01 & $\begin{array}{c}-0.01 \\
3.0 \times 10^{-3}\end{array}$ & -0.01 & $-1.0 \times 10^{-4}$ & $-3.0 \times 10^{-3}$ \\
\hline \multirow{3}{*}{$\begin{array}{l}\text { Moment of } \\
\text { ineritia }\end{array}$} & Thigh & -0.08 & -0.06 & -0.06 & -0.19 & -0.08 & -0.19 & 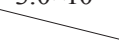 & 0.12 & & -0.03 \\
\hline & Shank & 0.42 & 0.34 & 0.13 & 0.95 & 0.63 & 1.27 & 0.77 & -0.76 & -0.01 & 0.13 \\
\hline & Foot & & 0.12 & & & & & & -0.56 & & \\
\hline
\end{tabular}

$\mathrm{p}$ values were all $<0.05$. The diagonal line meant that it was not included by statistical analysis.

Table 5. Correlation between predicted and actual joint moments

\begin{tabular}{|c|c|c|c|c|c|c|c|}
\hline & & \multicolumn{3}{|c|}{ AJM } & \multicolumn{3}{|c|}{ KJM } \\
\hline & & Extension peak & Flexion peak & $\mathrm{R}$ & Extension peak & Flexion peak & $\mathrm{R}$ \\
\hline \multirow{2}{*}{ Half squat } & Predicted & $13(3)$ & $-12(6)$ & \multirow{2}{*}{$0.34(0.39)$} & $55(10)$ & $-13(7)$ & \multirow{2}{*}{$0.93(0.03)$} \\
\hline & Actual & $19(6)^{*}$ & $-27(13)^{*}$ & & 75 (14)* & $-13(5)$ & \\
\hline \multirow{2}{*}{ Knee flexion } & Predicted & 24 (11) & $-205(24)$ & \multirow{2}{*}{$0.89(0.03)$} & $71(19)$ & $-7(6)$ & \multirow{2}{*}{$0.78(0.12)$} \\
\hline & Actual & $26(12)$ & $-161(41)^{*}$ & & $76(18)$ & $-27(21)^{*}$ & \\
\hline \multirow{2}{*}{ Knee extension } & Predicted & $483(71)$ & $-134(73)$ & \multirow{2}{*}{$0.94(0.02)$} & $242(61)$ & $-9(12)$ & \multirow{2}{*}{$0.94(0.03)$} \\
\hline & Actual & $413(54)^{*}$ & $-67(20)^{*}$ & & $233(28)$ & $-23(19)$ & \\
\hline \multirow{2}{*}{ Walking } & Predicted & $58(14)$ & $-14(10)$ & \multirow{2}{*}{$0.79(0.08)$} & 17 (3) & $-12(2)$ & \multirow{2}{*}{$0.51(0.15)$} \\
\hline & Actual & $78(10)^{*}$ & $-4(2)^{*}$ & & 17 (4) & $-52(17)^{*}$ & \\
\hline
\end{tabular}

$\mathrm{p}$ values were all $<0.001$. Each visible value showed mean $(\mathrm{SD})$.

* Indicated significant difference between groups $\mathrm{p}<0.05$.

of inertia differed by gender and calculated from the body weight and the leg lengths, the influence of gender and body type was considered minimum. Thus, angular information of the segment was central for joint moments estimation.

KJM had good to excellent correlation but AJM had fair correlation during Half squat. On the other hand, good to excellent correlations were observed for both AJM and KJM in Knee flexion-extension during single leg stance. These knee bending were used for calibrating when measuring kinematic in sagittal plane ${ }^{22}$. Since angular information was central in the prediction formulas, it is considered that sufficient estimation was possible with the calibrating motions that easily reflects the rotation of the segments.

Both AJM and KJM showed more than moderate to good correlations during Walking. The joint moments measurements in gait were diversified. For instance, Yang et al. ${ }^{23}$ ) added frontal and transverse intersegmental forces in addition to the kinematics of sagittal plane. Also, Karatsidis et al. ${ }^{24)}$ used distribution algorithm from 17 motion sensors. These studies showed good to excellent correlations (from $\mathrm{R}=0.8$ ), which indicates that intersegmental forces other than sagittal plane or many more sensors are required to obtain higher correlations.

Peak values in each direction of the predicted moments had some differences between actual ones. However, pursuing the accuracy makes it difficult to use in the clinics as more factors should be considered or more sensors are required ${ }^{23,24)}$. Kodama et al. ${ }^{10)}$ required at least total 32 parameters in both horizontal and vertical for estimation of joint moments. However, the prediction formulas in this study created from maximum 9 parameters using only three sensors and can obtain good to excellent correlations between actual joint moments for most of the motions. Thus, it is suggested that angle information of the segment CoM and moments of inertia can provide quantitative and time-based estimation for joint moments. 
There are several limitations in the prediction formulas. Firstly, joint moments cannot be measured with the same accuracy as the large scale devices. Secondly, the sample size was small and limited to young healthy adults. Lastly, only AJM and KJM in sagittal plane were assumed, not including joint moments for other joints or in other than sagittal plane.

Further studies are necessary so that put to practical use in busy clinics. All data used for the prediction formulas were cut off to 30 samples per second, which is the same sampling frequency as the normal video camera ${ }^{25,26)}$. This means the acquisition of angular information could be substituted from the movie ${ }^{27)}$. Recently, smartphones, which equipped with tri-axial accelerometer, gyroscopes and magnetic sensors, are widespread and can be used in place of the motion sensors ${ }^{28)}$. These tools could be more clinically useful and need to consider whether can be used to estimate joint moment. Furthermore, an electromechanical delay exists from the onset of muscle electrical activation to the onset of joint moment ${ }^{29)}$. Incorporating EMG could be more useful as an assessment of the timing of muscle strength exertion during motions.

In conclusion, it is suggested that the prediction formulas created from angle information of the segment CoM and moments of inertia can be used for approximate estimation of lower limb joint moments in sagittal plane. And more clinically useful tools need to consider in the future.

\section{Presentation at a Conference}

This paper was presented in World Confederation for Physical Therapy 2019 in Geneva.

http://www.professionalabstracts.com/wcpt2019/iplanner/\#/list

\section{Funding and Conflict of interest}

There are no funding and conflicts of interest to this research.

\section{REFERENCES}

1) Lu TW, Hsu HC, Chang LY, et al.: Enhancing the examiner's resisting force improves the reliability of manual muscle strength measurements: comparison of a new device with hand-held dynamometry. J Rehabil Med, 2007, 39: 679-684. [Medline] [CrossRef]

2) Moriello C, Mayo NE: Development of a position-specific index of muscle strength to be used in stroke evaluation. Arch Phys Med Rehabil, 2006, 87: 14901495. [Medline] [CrossRef]

3) Sultan SM: The assessment and importance of disease activity versus disease damage in patients with inflammatory myopathy. Curr Rheumatol Rep, 2003, 5: 445-450. [Medline] [CrossRef]

4) Chamorro C, Armijo-Olivo S, De la Fuente C, et al.: Absolute reliability and concurrent validity of hand held dynamometry and isokinetic dynamometry in the hip, knee and ankle joint: systematic review and meta-analysis. Open Med (Wars), 2017, 12: 359-375. [Medline]

5) Veerbeek JM, van Wegen E, van Peppen R, et al.: What is the evidence for physical therapy poststroke? A systematic review and meta-analysis. PLoS One, 2014, 9: e87987. [Medline] [CrossRef]

6) Beggs JS: Kinematics. USA: Hemisphere Pub, 1983, p 1.

7) Astephen JL, Deluzio KJ, Caldwell GE, et al.: Gait and neuromuscular pattern changes are associated with differences in knee osteoarthritis severity levels. J Biomech, 2008, 41: 868-876. [Medline] [CrossRef]

8) Eitzen I, Fernandes L, Nordsletten L, et al.: Weight-bearing asymmetries during Sit-To-Stand in patients with mild-to-moderate hip osteoarthritis. Gait Posture, 2014, 39: 683-688. [Medline] [CrossRef]

9) Zijlstra W, Bisseling R: Estimation of hip abduction moment based on body fixed sensors. Clin Biomech (Bristol, Avon), 2004, 19: 819-827. [Medline] [CrossRef]

10) Kodama J, Watanabe T: Examination of inertial sensor-based estimation methods of lower limb joint moments and ground reaction force: results for squat and sit-to-stand movements in the sagittal plane. Sensors (Basel), 2016, 16: E1209. [Medline] [CrossRef]

11) Ae M, Tang H, Yokoi T: Estimation of inertia properties of the body segments in Japanese athletes. Biomechanism, 1992, 11: 23-33 (in Japanese). [CrossRef]

12) Debray TP, Vergouwe Y, Koffijberg H, et al.: A new framework to enhance the interpretation of external validation studies of clinical prediction models. J Clin Epidemiol, 2015, 68: 279-289. [Medline] [CrossRef]

13) Choo MS, Jeong SJ, Cho SY, et al.: Development of decision support formulas for the prediction of bladder outlet obstruction and prostatic surgery in patients with lower urinary tract symptom/benign prostatic hyperplasia: part II, external validation and usability testing of a smartphone app. Int Neurourol J, 2017, 21: S66-S75. [Medline] [CrossRef]

14) Anderson DE, Madigan ML, Nussbaum MA: Maximum voluntary joint torque as a function of joint angle and angular velocity: model development and application to the lower limb. J Biomech, 2007, 40: 3105-3113. [Medline] [CrossRef]

15) Dionisio VC, Almeida GL, Duarte M, et al.: Kinematic, kinetic and EMG patterns during downward squatting. J Electromyogr Kinesiol, 2008, 18: 134-143. [Medline] [CrossRef]

16) Fong DT, Chan YY: The use of wearable inertial motion sensors in human lower limb biomechanics studies: a systematic review. Sensors (Basel), 2010, 10: 11556-11565. [Medline] [CrossRef]

17) Teruyama $Y$, Watanabe T: Effectiveness of variable-gain Kalman filter based on angle error calculated from acceleration signals in lower limb angle measurement with inertial sensors. Comput Math Methods Med, 2013, 2013: 398042. [Medline] [CrossRef]

18) Coley B, Najafi B, Paraschiv-Ionescu A, et al.: Stair climbing detection during daily physical activity using a miniature gyroscope. Gait Posture, 2005, 22: 287-294. [Medline] [CrossRef]

19) Liu SH, Lin CB, Chen Y, et al.: An EMG patch for the real-time monitoring of muscle-fatigue conditions during exercise. Sensors (Basel), 2019 , 19: E3108. [Medline] [CrossRef] 
20) Alvarez JC, Álvarez D, López AM: Accelerometry-based distance estimation for ambulatory human motion analysis. Sensors (Basel), 2018, 18: E4441. [Medline] [CrossRef]

21) Portney LG, Watkins MP: Clinical foundations of clinical research. applications to practice, 3rd ed. Upper Saddle River: Pearson Education, 2009 , p 525.

22) Ferrari A, Cutti AG, Garofalo P, et al.: First in vivo assessment of "Outwalk": a novel protocol for clinical gait analysis based on inertial and magnetic sensors. Med Biol Eng Comput, 2010, 48: 1-15. [Medline] [CrossRef]

23) Yang EC, Mao MH: 3D analysis system for estimating intersegmental forces and moments exerted on human lower limbs during walking motion. Measurement, 2015, 73: 171-179. [CrossRef]

24) Karatsidis A, Bellusci G, Schepers HM, et al.: Estimation of ground reaction forces and moments during gait using only inertial motion capture. Sensors (Basel), 2016, 17: E75. [Medline] [CrossRef]

25) Ehlert S, Kingery A, Suggs R: Improving photometric calibration of meteor video camera systems. Planet Space Sci, 2017, 143: 218-224. [Medline] [CrossRef]

26) Martindale CF, Hoenig F, Strohrmann C, et al.: Smart annotation of cyclic data using hierarchical hidden Markov models. Sensors (Basel), 2017, 17: E2328. [Medline] [CrossRef]

27) Finkbiner MJ, Gaina KM, McRandall MC, et al.: Video movement analysis using smartphones (ViMAS): a pilot study. J Vis Exp, 2017, 14. [Medline]

28) Mourcou Q, Fleury A, Franco C, et al.: Performance evaluation of smartphone inertial sensors measurement for range of motion. Sensors (Basel), 2015, 15: 23168-23187. [Medline] [CrossRef]

29) Cavanagh PR, Komi PV: Electromechanical delay in human skeletal muscle under concentric and eccentric contractions. Eur J Appl Physiol Occup Physiol, 1979, 42: 159-163. [Medline] [CrossRef] 